\title{
Title:
}

\section{Thiopurine adherence: high prevalence with low impact in UC outcomes}

\section{Authors:}

Concepción Gómez-Medina, María Capilla-Lozano, María Pilar Ballester Ferré, David Martí-Aguado, Ana Crespo, Marta Maia Bosca-Watts, Pablo Navarro Cortés, Rosario Antón, Isabel Pascual Moreno, Joan Tosca Cuquerella, Miguel Mínguez Pérez

DOI: $10.17235 /$ reed.2021.7630/2020

Link: PubMed (Epub ahead of print)

Please cite this article as:

Gómez-Medina Concepción, Capilla-Lozano María, Ballester Ferré María Pilar, Martí-Aguado David, Crespo Ana, Bosca-Watts Marta Maia, Navarro Cortés Pablo, Antón Rosario, Pascual Moreno Isabel, Tosca Cuquerella Joan, Mínguez Pérez Miguel. Thiopurine adherence: high prevalence with low impact in UC outcomes . Rev Esp Enferm Dig 2021. doi: 10.17235/reed.2021.7630/2020.

This is a PDF file of an unedited manuscript that has been accepted for publication. As a service to our customers we are providing this early version of the manuscript. The manuscript will undergo copyediting, typesetting, and review of the resulting proof before it is published in its final form. Please note that during the production process errors may be discovered which could affect the content, and all legal disclaimers that apply to the journal pertain. 


\section{OR 7630}

\section{Thiopurine adherence: high prevalence with low impact in UC outcomes}

Concepción Gómez-Medina ${ }^{1}$, María Capilla-Lozano ${ }^{1}$, María Pilar Ballester Ferré ${ }^{1}$, David Martí-Aguado $^{1}$, Ana Crespo ${ }^{2}$, Marta Maia Bosca-Watts ${ }^{1}$, Pablo Navarro Cortés ${ }^{1}$, Rosario Antón ${ }^{1}$, Isabel Pascual Moreno ${ }^{1}$, Joan Tosca Cuquerella ${ }^{1}$ and Miguel Minguez Pérez ${ }^{1}$. From the ${ }^{1}$ Clinic University Hospital of Valencia; ${ }^{2}$ University of Valencia.

Correspondence: Concepción Gómez-Medina conchin.gome@gmail.com

Authors' contributions:

Conception and design of the study: Gómez-Medina C, Capilla-Lozano M, Ballester MP, Martí-Aguado D, Crespo A, and Minguez M.

Acquisition of data: Gómez-Medina C, Capilla-Lozano M, Ballester MP, Martí-Aguado D, Crespo A., Navarro P, Bosca-Watts MM, Tosca J, Minguez M.

Analysis and interpretation of data: Gómez-Medina C, Capilla-Lozano M, Ballester MP, Martí-Aguado D, Crespo A, and Minguez M.

Manuscript writing: Gómez-Medina C, Capilla-Lozano M, Ballester MP, Martí-Aguado D, Bosca-Watts MM and Minguez $\mathrm{M}$.

Manuscript review: Gómez-Medina C, Capilla-Lozano M, Ballester MP, Martí-Aguado D, Bosca-Watts MM, Navarro P, Antón R, Pascual I, Tosca J and Minguez M.

All listed authors approved the final version.

Compliance with ethical standards

Conflict of interest M. Minguez has served as a speaker, a consultant, and advisory member for or has received research funding from MSD, Abbvie, Pfizer, Janssen, Shire Pharmaceuticals, Allergan.

M.M. Bosca-Watts declares educational activities, research projects, scientific meetings, and advisory boards sponsored by MSD, Ferring, Abbvie, Janssen, and Takeda.

Informed consent statement: All study participants, or their legal guardian, provided informed written consent prior to study enrollment. 
Institutional review board approval. The study was reviewed and approved by the University Clinic Hospital of Valencia, Institutional Review Board.

Acknowledgement: Ballester MP and Marti-Aguado D (CM19/00212) are both recipient of a Río Hortega award, Instituto de Salud Carlos III.

Summary of the article's main point: Thiopurines are used as maintenance therapy in patients with ulcerative colitis, adherence to treatment is supposed to play an important role. In our cohort of patients, adherence to thiopurines is high and is not related to clinical or pharmacological factors.

\begin{abstract}
Introduction

Thiopurines are used as maintenance therapy in patients with ulcerative colitis (UC). There are contradictory results regarding the relationship between adherence to treatment and risk of relapse.
\end{abstract}

\title{
Objectives
}

To quantify and evaluate the trends in thiopurines prescription rates, and to determine the impact and risk factors of non-adherence.

\section{Methods}

Analytical, observational and retrospective study of UC patients, on thiopurines, included in the ENEIDA single-center registry from October 2017 to October 2019. We included adult patients under clinical remission at the beginning of the study on thiopurines maintenance treatment for at least 6 months before recruitment. Adherence was evaluated with an electronic pharmaceutical prescription system. Adherence was considered when $80 \%$ or more of the prescribed medication was dispensed at the pharmacy. Kaplan-Meier curves and a regression model were used to examine year-toyear treatment dispensation and identify factors associated to non-adherence.

\section{Results}

A total of 41 patients were included, of whom $71 \%$ were males with a mean age of 44 (?14) and $26.8 \%$ were concomitantly managed with biological therapy. Overall, $22 \%$ were 
non-adherent to thiopurines. No predictive factors of non-adherence were identified. Adherence rate did not correlate with disease activity for two years follow-up (OR 1.6; $95 \mathrm{Cl}=0.3-9.1)$. Left-sided colitis and concomitant biological treatment were related with disease relapses $(p \leq 0.01)$.

\section{Conclusion}

The adherence to thiopurines in UC patients is high (78\%). Non-adherence is not related to clinical or pharmacological factors. Adherence rate was not associated with disease activity.

Keywords Colitis, ulcerative. Thiopurines . Treatment adherence and compliance . Treatment outcome.

\section{Introduction}

Inflammatory bowel disease (IBD) is a chronic disease characterized by the alternation of flares and remissions. Thiopurines (azathioprine or 6-mercaptopurine) are long-term treatments used to maintain steroid-free remission in corticoid-dependent patients and to decrease the immunogenicity of biological drugs.

Effectiveness of the treatment depends, among other clinical, analytical and biological factors, on adherence, with a clear correlation between less adherence and worse evolution of the disease and/or higher risk of relapse (1-4). However, other studies have not found an impact of adherence to immunomodulators on disease outcomes (5-12). These contradictory results can be explained by two reasons: the absence of a well-defined limit for non-adherence and the wide variety of methods used to measure it.

Studies using an electronic pharmaceutical system employ a cut-off of $80 \%(1,6-9)$ to define adherence while others set the limit at 95\% (13-14). Published rates of treatment non-adherence in IBD range from 7 to $72 \%$, although most studies describe rates of $30-40 \%$ (9). Focusing exclusively on thiopurines, non-adherence varies between 7 and $64 \%$ (5-7, 10-12, 15-20); nevertheless, studies do not distinguish between UC and CD which can be by itself a confounding factor $(11,13,21)$.

To assess thiopurine adherence, direct (measure metabolites in blood or urine) and indirect methods (questionnaires, clinical interviews, pharmacy data) are used. The electronic pharmaceutical system is an objective indirect method that analyses the 
proportion of medication dispensed in pharmacy with respect to what was prescribed. It has been shown to have a good correlation with thiopurine adherence obtained using the Morisky Medication Adherence 8-item Scale (MMAS-8) $(19,22)$, an eight-item validated survey derived from the Morisky scale that assesses treatment adherence. Likewise, this method also correlates with the serum or urine drug levels, as well as with the effect of drug treatment on the disease $(20,23-24)$. When analyzing risk factors for non-adherence, not only patient and disease related factors are relevant, but also social environment and healthcare system conditions (equity, accessibility, waiting time, costs, drug storage) (25). One study carried out by de Castro et al. (19) uses the electronic prescription system to evaluate adherence in our health system. Nevertheless, none of the previous mentioned studies evaluated the impact of adherence in the evolution of the disease by an electronic prescription system within a public health system.

Given that the actual impact of adherence to thiopurines on the risk of flares is unclear, we believe that performing a study with an objective, cheap and easy-to-manage method in a real-life scenario will allow us to properly assess the percentage of adherence and therefore to evaluate the effect of non-adherence on disease outcomes.

We hypothesized that despite a high adherence ratio of our population study there is no direct impact on the evolution of the disease. So, the main aim of this study was to analyze, using an electronic pharmaceutical prescription system, thiopurine adherence. Secondary aims were to identify risk factors associated with non-adherence and its impact on the risk of flares in patients with UC controlled in our IBD Unit.

\section{Material and methods}

\section{$\underline{\text { Study design and population }}$}

An observational, retrospective, single tertiary center, cohort study was carried out. All consecutive patients with UC followed in the IBD Unit of the Clinic University Hospital of Valencia were screened for eligibility. Inclusion criteria were firm UC diagnosis, age $\geq 18$ years old, clinical remission at the beginning of the study, maintenance treatment with thiopurines (azathioprine or 6-mercaptopurine) at least 6 months before the study and throughout the whole period of the study, and to have a prescription made with the pharmaceutical electronic program and signed informed consent. Exclusion criteria were patient history of colon surgery, intolerance or hypersensitivity to 
thiopurines and/or loss of follow-up during the study period.

Patient, disease and therapy data were collected from the ENEIDA registry at the beginning of the study. ENEIDA is a registry of the Spanish Working Group in Crohn's and Colitis (GETECCU), which includes patients with IBD and records prospectively clinical characteristics, outcomes, and treatments. To assess adherence and flares, electronic medical records were reviewed during two-years follow-up (from October 2017 to October 2019). Adherence to treatment was evaluated with a pharmaceutical electronic management program. With this application, the percentage of the prescribed doses of thiopurines that the patient dispensed at the pharmacy was calculated. Adherence time was estimated until relapse or the end of the study period in cases under remission. Patients who picked up $80 \%$ or more of the prescribed medication from the pharmacy were defined as adherent (3-7).

Informed consent to participate in the database was obtained from all patients. The study was approved by the institutional ethics committee of the hospital on July 24 , 2018. The study protocol conforms to the ethical guidelines of the 2013 Declaration of Helsinki as reflected in a priori approval by the institution's human research committee.

\section{Definitions}

The diagnosis of UC was made through clinical, radiological, endoscopic and histopathological criteria according to the European Crohn's and Colitis Organization (ECCO) consensus guidelines (26). Disease extent was determined with ileocolonoscopy and classified according to the Montreal classification (27). The maximum extent of the disease at any time since diagnosis was assigned for each patient.

Family history of IBD was defined as the presence of one or more first or seconddegree relatives with either UC or CD. Extraintestinal manifestations (EIM) included periphery arthropathy, ankylosing spondylitis, sacroileitis, skin or ocular manifestation, primary sclerosing cholangitis (PSC) and stomatitis. Megacolon, massive hemorrhage, perforation or intraabdominal abscess were considered as complications of the disease.

Concomitant treatment related and not-related with IBD (including allopurinol separately) was analyzed at the beginning of the study so that patients which were prescribed treatment before October 2017 and through all the study period were considered. Chronic (hypertension, diabetes mellitus and dyslipidemia) and psychiatric 
pathologies (anxiety and depression) were also analyzed as qualitative variables and recorded according to the international classification of diseases (ICD) (28), when they were specifically recorded in the medical history of the patient.

A mild relapse was considered as the presence of active disease symptoms that required treatment modification: increase in the dosage of 5-aminosalicylic acid (5-ASA) or 5-ASA combination (oral + topical administration). Moderate-severe relapse was defined by the need for treatment with corticosteroids (topic or systemic action) and/or biological drugs and/or surgery for active disease. We calculated the partial Mayo index at the beginning, the sixth month, one year and the second year after the beginning of the study to evaluate the evolution of the disease throughout the follow-up.

\section{Statistical analysis}

Quantitative variables were expressed as mean and standard deviation (SD) or as median and interquartile range (IQR) whether they followed or not a normal distribution. Qualitative variables were expressed as frequencies (\%). Univariate analysis was performed to determine variables associated with non-adherence. Categorical variables were compared using the Chi-Square or Fisher's test. Measures of association were reported as odds ratio (OR) with $95 \%$ confidence intervals $(95 \mathrm{Cl})$. Quantitative variables were analyzed with the Student $t$ test, Mann-Whitney $U$ test or Kruskal-Wallis test, as required. A multivariate logistic regression analysis including only the significant factors of the univariate analysis was made to identify independent risk factors of non-adherence. $A$ Spearman's rank correlation analysis was performed to associate percentage of adherence with relapse rates. Time to relapse was analyzed using Kaplan-Meier curves. Survival curves were compared using log-rank test to identify factors associated with disease activity during follow up. Statistical analysis was performed with the IBM SPSS Statistics 25 program and $p$-values $<0.05$ were considered significant

\section{Results}

\section{Study population and prevalence of adherence}

Of a total of 764 patients with UC registered in the ENEIDA database, 218 (29\%) had been treated with thiopurines. Of those, 82 (37.6\%) patients were under treatment in October 2017, median Partial Mayo Index of 0 (0-0). Applying the inclusion and exclusion criteria, a total of 41 patients were finally enrolled in the study (Figure 1). Patients 
baseline characteristics are described in Table 1.

The indication for thiopurines in our cohort of patients was mainly for maintenance of remission (80.5\%), association with biological treatment (14.6\%), EIM $(2.4 \%)$ and fistulizing disease $(2.4 \%)$ being $36.6 \%$ corticodependent. $19.5 \%(n=8)$ of our patients suffered from an adverse event to thiopurines, being leucopenia the most frequent one $(75 \%)$.

Overall, $78 \%$ of study population ( $n=32$ ) were adherent to thiopurines during the two years follow-up. Median dispensation rate was 96\% (89-100) among adherent group and 68\% (44-75) among non-adherent group ( $p<0.001)$. Importantly, focusing on the nonadherent group $(n=9$ ), only two subjects had a dispensation rate below $70 \%$ and $44 \%$ were under biological treatment. Adherence to biological therapy was also estimated, and none were non-adherent.

\section{$\underline{\text { Risk factors for non-adherence }}$}

No demographic, phenotypic factors of the disease or therapeutic regimens were predictors of the thiopurine non-adherence condition (Table 2).

Risk factors for disease flare

Over two years follow-up there were 12 patients $(29.3 \%)$ who suffered from a disease relapse being $19.5 \%(n=8)$ mild and $14.6 \%(n=6)$ moderate-severe, two patients suffered from mild and moderate-severe relapse. The median time to disease relapse was 15 (4-54) weeks, 12 (4-50) weeks in the mild relapse and 24 (4-54) weeks in the moderatesevere relapse. The indication of thiopurines showed no statistically relationship with adherence nor disease relapse.

No statistically significant relationship was observed between adherence to treatment, considering $80 \%$ as cut-off, and activity relapses, with $22.2 \%$ relapses in nonadherent vs $31.3 \%$ relapses in adherent (OR $1.6 ; 95 \mathrm{Cl}=0.28-9.07 ; p=0.7)$, nor with mild (OR 2.24; $95 \mathrm{Cl}=0.24-21.07 ; p=0.66)$ or moderate-severe $(\mathrm{OR} 0.5 ; 95 \mathrm{Cl}=0.08-3.3 ; p=0.6)$ relapses, when analyzed separately. No difference was found in Partial Mayo Index between adherent and non-adherent group through the whole study period, median score of $0(0-0)$.

The Spearman's rank correlation analysis showed a positive coefficient (0.036) between adherence and the relapse rates but with no statically relationship $(p=0.82)$. The 
cumulative proportion of relapse at week 60 in the non-adherent group was $22 \%$ vs $28 \%$ in the adherent group. These differences were not statistically significant (log Rank $=0.37$, $p=0.543)$. (Figure 2)

Independently from the adherence factor, we observed significant relationship between diseases flares and other variables (Table 2). A higher risk of relapse was associated with left-sided colitis (OR 8.67; $95 \mathrm{Cl}=1.67-44.94 ; p=0.01)$; the cumulative proportion of relapse at week 60 in the proctitis group was $0 \%, 56 \%$ in the left-sided colitis group and $20 \%$ in the pancolitis group with statistically significant relation between groups $(p=0.015, \log$ Rank $=8.41)$ (Figure 3). Moreover, a higher risk of disease relapse was observed in patients who were prescribed a biological treatment $(\mathrm{OR} 8.75 ; 95 \mathrm{Cl}=$ $1.84-41.61 ; p=0.007)$.

In the multivariate analysis, the mentioned variables were statistically significant, presence of left-sided colitis $(\mathrm{HR}=5.6 ; 95 \mathrm{Cl}=1.61-19.47 ; p=0.007)$ and treatment with biologics $(\mathrm{HR}=0.139 ; 95 \mathrm{Cl}=0.04-0.5 ; p=002)$, remaining adherence as a non-independent factor for disease relapse $(\mathrm{HR}=0.195 ; 95 \mathrm{Cl}=0.04-1.06 ; p=0.06)$.

\section{Discussion}

We observed that with an electronic pharmaceutical management system, thiopurine non-adherence was seen in approximately one out of four patients with UC without an independent significant relationship with disease flares during 2 years followup.

Our study showed a median possession rate of $94 \%$ (81-100), when considering adherence as a quantitative variable. The non-adherence rate was $22 \%$ when considering it as a qualitative variable, that is, within the wide range of 7 to $64 \%$ described in previous studies $^{19-20}$.

We chose the electronic pharmaceutical prescription system of the Valencian Community as an objective and indirect procedure to assess adherence in order to avoid the subjectivity of self-reported questionnaires and the motivation for adherence to treatment that can be seen with direct methods. That can explain the non-adherence higher rate showed in our study compared to that documented in studies that measure adherence through the analysis of metabolites (10-17\%) $(5-6,11,20)$ and self-reported questionnaires $(7-15 \%)(6,16-17,20)$. With the electronic prescription system, patients 
were blinded to the methodology and, therefore, no change on patients' attitude could modify results, reflecting a real clinical practice situation. In addition, this method has a high specificity to identify non-adherent patients because medication in this healthcare area cannot be obtained from sources other than the pharmacy (29). It must be taken into account that this method does not guarantee that the medication was actually taken and therefore the percentage of adherence may have been overestimated. However, there are studies that state that the prescribed medication dispensing rates are quite accurate to calculate adherence when performed in a healthcare system with electronic treatment records and with a closed pharmaceutical system (14). There is no gold standard to assess adherence $(14,30)$ and different methods should be combined.

The results of this study showed that non-adherence to thiopurines was not an independent predictive factor for disease relapse when analyzed as a dichotomy variable, taking into account $80 \%$ as a cut-off and as a quantitative variable without finding a significant cut-off for defining adherence. This fact could be justified by the low relapse rate found in our cohort of patients. No differences in the Partial Mayo Index through the study period were found, with a median score of $0(0-0)$. So, patients in our study were mainly in clinical remission and were at treatment with thiopurines for maintenance of remission (80.5\%), this could explain the low relapse rate compared to other studies. However, we did not analyze the time that patients have been in remission before being included in the study, since if they are patients with a long-time remission, the probability of relapse may be less.

Regarding the type of drug used, adherence to thiopurines (78\%) was higher than adherence to 5-ASA (59\%) for patients in our cohort who were treated with both medications during the first year follow-up, in line with previously analyzed data in the same database (31-32), and other articles in the medical literature $(6,15,17)$. On the other hand, those patients who received treatment with biological drugs showed $100 \%$ adherence. This finding is probably due to the fact that these drugs are used in more complex stages of the disease in which disease awareness is higher, and most of them were administered intravenously, in a healthcare environment (21). The way the drug is administered (subcutaneously or intravenously of anti-TNF versus oral administration of 5ASA and thiopurines) and the lower dosing frequency should also be taken into account, 
because patients on oral therapy have to be adherent to a daily administered treatment.

The demographic characteristics of the patients (gender and age) were not significantly associated with the lack of adherence to thiopurines. Although the lack of adherence has been classically linked to young age $(2,13,33)$ and males $(1,7,12)$, other studies also describe the absence of significant differences between these variables and adherence to treatment in $\operatorname{IBD}(6,9,16,17,19)$. Similarly, the clinical disease characteristics of our patients did not show statistically significant differences to establish themselves as a predictive factor of adherence. The comorbidity of the patients was also evaluated in the study. We analyzed chronic and psychiatric pathologies, variables that had been previously considered in the medical literature, but we did not find significant differences for any of these variables. We did observe a tendency towards lower adherence in patients with depression than those who suffered from anxiety or other comorbidities.

Independently from the adherence factor, we found a statistically significant relationship between a higher risk of disease relapses with the presence of left-sided colitis and the prescription of concomitant treatment with a biological drug. Those patients in treatment with biological therapy are supposed to be in more advanced stages of the disease and therefore have a higher risk of disease relapse.

The main limitation of our study was the small sample size that could have conditioned the statistical power of the study. We suggest studies with a greater number of patients, which incorporate a second method of adherence evaluation to give greater validity to the results. Other limitation for the survival analysis was not to include the patients who discontinue treatment during the study period, they could have been included during active treatment and censored at the end, in order to know the impact in more detail. It could also be interesting to study other causes of non-adherence, such as socioeconomic or employment status, as well as to know if adherence to therapy varies throughout the time of prescription of the drug.

In conclusion, $78 \%$ of UC patients in clinical remission evaluated for two years are adherent to thiopurines, with a median possession rate of $94 \%$ through a prescription electronic program. No demographic (age, gender), phenotypic factors of the disease (extension, time of evolution, family association, comorbidity) or therapeutic regimens 
were predictors of the thiopurine non-adherence condition. Moreover, no significant impact on the evolution of the disease was shown, probably due to the low relapse rate and the main indication of thiopurines (maintenance of remission). When analyzing other variables different from adherence (extension and biological treatment), a statistically significant relationship was found with disease relapses. It may be necessary to carry out studies with a larger sample size to evaluate the actual impact of the adherence to thiopurines in the evolution of the disease.

\section{References}

1. Robinson A. Review article: improving adherence to medication in patients with inflammatory bowel disease. Aliment Pharmacol Ther. 2008;27(1):9-14.

2. Trindade AJ, Ehrlich A, Kornbluth A, et al. Are your patients taking their medicine? Validation of a new adherence scale in patients with inflammatory bowel disease and comparison with physician perception of adherence. Inflamm Bowel Dis. 2011;17(2):599-604.

3. Kane S, Huo D, Aikens J, et al. Medication Nonadherence and the Outcomes of Patients with Quiescent Ulcerative Colitis. Am J Med. 2003;114(1):39-43

4. Kane SV. Systematic review: adherence issues in the treatment of ulcerative colitis. Aliment Pharmacol Ther. 2006;23(5):577-85.

5. Gilissen LPL, Wong DR, Engels LGJB, et al. Therapeutic drug monitoring of thiopurine metabolites in adult thiopurine tolerant IBD patients on maintenance therapy. J Crohns Colitis. 2012;6(6):698-707.

6. Bokemeyer B, Teml A, Roggel C, et al. Adherence to thiopurine treatment in outpatients with Crohn's disease. Aliment Pharmacol Ther. 2007;26(2):217-25.

7. Mantzaris GJ, Roussos A, Kalantzis C, et al. How Adherent to Treatment with 
Azathioprine Are Patients with Crohn's Disease in Long-term Remission? Inflamm Bowel Dis. $2007 ; 13(4): 446-50$.

8. Ediger JP, Walker JR, Graff L, et al. Predictors of Medication Adherence in Inflammatory Bowel Disease. Am J Gastroenterol. 2007;102(7):1417-26.

9. Hommel KA, Davis CM, Baldassano RN. Medication adherence and quality of life in pediatric inflammatory bowel disease. J Pediatr Psychol. 2008;33(8):867-74.

10. LeLeiko NS, Lobato D, Hagin S, et al. 6-Thioguanine Levels in Pediatric IBD Patients. Inflamm Bowel Dis. 2013;19(12):2652-8.

11. Kamperidis N, Goodhand JR, Chowdhury FA, et al. Factors Associated With Nonadherence to Thiopurines in Adolescent and Adult Patients With Inflammatory Bowel Disease. J Pediatr Gastroenterol Nutr. 2012;54(5):685-9.

12. Campos S, Portela F, Sousa P, et al. Inflammatory bowel disease: adherence to immunomodulators in a biological therapy era. Eur J Gastroenterol Hepatol. 2016;28(11):1313-9.

13. $D^{\prime}$ incà $R$, Bertomoro $P$, Mazzocco $K$, et al. Risk factors for non-adherence to medication in inflammatory bowel disease patients. Aliment Pharmacol Ther. 2007;27(2):166-72.

14. Osterberg L, Blaschke T. Adherence to Medication. N Engl J Med. 2005;353(5):487-97.

15. Oliva-Hemker MM, Abadom V, Cuffari C, et al. Nonadherence with Thiopurine Immunomodulator and Mesalamine Medications in Children with Crohn Disease. J Pediatr Gastroenterol Nutr. 2007;44(2):180-4.

16. Hommel KA, Davis CM, Baldassano RN. Objective versus subjective assessment of oral medication adherence in pediatric inflammatory bowel disease. Inflamm Bowel Dis. 2009;15(4):589-93.

17. Hommel KA, Hente EA, Odell S, et al. Evaluation of a group-based behavioral intervention to promote adherence in adolescents with inflammatory bowel disease. Eur J Gastroenterol Hepatol. 2012;24(1):64-9.

18. LeLeiko NS, Lobato D, Hagin S, et al. Rates and Predictors of Oral Medication Adherence in Pediatric Patients with IBD. Inflamm Bowel Dis. 2013;19(4):832-9.

19. de Castro ML, Sanromán L, Martín A, et al. Assessing medication adherence in 
inflammatory bowel diseases. A comparison between a self-administered scale and a pharmacy refill index. Rev Esp Enferm Dig. 2017;109(8):542-51.

20. Selinger CP, Ochieng AO, George V, et al. The Accuracy of Adherence Self-report Scales in Patients on Thiopurines for Inflammatory Bowel Disease: A Comparison With Drug Metabolite Levels and Medication Possession Ratios. Inflamm Bowel Dis. 2018;0(0):1-6.

21. Lopez, A., Billioud, V., Peyrin-Biroulet, C., et al. (2013). Adherence to Anti-TNF Therapy in Inflammatory Bowel Diseases. Inflamm Bowel Dis., 19(7), 1528-1533.

22. Kane S, Becker B, Harmsen WS, et al. Use of a screening tool to determine nonadherent behavior in inflammatory bowel disease. Am J Gastroenterol. 2012;107(2):154-60.

23. Bond CA, Monson R. Sustained improvement in drug documentation, compliance, and disease control. A four-year analysis of an ambulatory care model. Arch Intern Med. 1984;144(6):1159-62.

24. Steiner JF, Koepsell TD, Fihn SD, et al. A general method of compliance assessment using centralized pharmacy records. Med Care. 1988;26(8):814-23.

25. Marco Vincenzo Lenti \& Christian P. Selinger (2017) Medication non-adherence in adult patients affected by inflammatory bowel disease: a critical review and update of the determining factors, consequences and possible interventions, Expert Review of Gastroenterology \& Hepatology, 11:3, 215-226

26. Stange EF, Travis SPL, Vermeire S, et al. European evidence-based consensus on the diagnosis and management of ulcerative colitis: definitions and diagnosis. J Crohn's Colitis. 2008; 2:1-23.

27. Silverberg M, Satsangi J, Ahmad T, et al. Toward and integrated clinical, molecular, and serological classification of inflammatory bowel disease: re-port or a working party of the Montreal World Congress of Gastroenterology. Can J Gastroenterol 2005;19(Suppl A):5A-36A.

28. World Health Organization. (1978)|. International classification of diseases: [9th] ninth revision, basic tabulation list with alphabetic index. World Health Organization. https://apps.who.int/iris/handle/10665/39473 .

29. Andrade SE, Kahler $\mathrm{KH}$, Frech F, et al. Methods for evaluation of medication 
adherence and persistence using automated databases. Pharmacoepidemiol Drug Saf. 2006;15(8):565-74.

30. Jackson CA, Clatworthy J, Robinson A, et al. Factors Associated With NonAdherence to Oral Medication for Inflammatory Bowel Disease: A Systematic Review. Am J Gastroenterol. 2009;105(3):525-39.

31. Ballester, M. P., Marti-Aguado, D., Fullana, M., et al. (2019). Impact and risk factors of non-adherence to 5-aminosalicylates in quiescent ulcerative colitis evaluated by an electronic management system. Int J Colorectal Dis, 34(6), 1053-1059.

32. Marti-Aguado, D., Ballester, M. P., Tosca, J., et al. (2019). Long-term follow-up of patients treated with aminosalicylates for ulcerative colitis: Predictive factors of response: An observational case-control study. United European Gastroenterol J, 7(8), 1042-1050.

33. Tae $\mathrm{CH}$, Jung S-A, Moon HS, et al. Importance of Patients' Knowledge of Their Prescribed Medication in Improving Treatment Adherence in Inflammatory Bowel Disease. J Clin Gastroenterol. 2016;50(2):157-62.

Table 1. Basal population characteristics.

Basal characteristics

\begin{tabular}{l|l}
\hline $\begin{array}{l}\text { Gender } \\
\text { Male, } n \text { (\%) }\end{array}$ & $29(70.7)$ \\
$\quad$ Female, $n$ (\%) & $12(29.3)$ \\
Family history of IBD, $n$ (\%) & $8(19.5)$ \\
Smoking habit, $n$ (\%) & $4(9.8)$ \\
Appendectomy, $n$ (\%) & $2(4.9)$ \\
Disease extension, $n$ (\%) & \\
E1, proctitis & $2(4.9)$ \\
E2, left-sided colitis & $9(22)$ \\
E3, pancolitis & $30(73.2)$ \\
EIM, $n$ (\%) & $13(31.7)$ \\
Complications, $n$ (\%) & $5(12.2)$ \\
ADR to thiopurines, $n$ (\%) & $8(19.5)$
\end{tabular}

TOTAL POPULATION $(n=41)$ 


\begin{tabular}{l|l} 
Concomitant treatment related to IBD, $n$ (\%) & $30(73.2)$ \\
5-ASA treatment, $n$ (\%) & $30(73.2)$ \\
Biological treatment, $n$ (\%) & $11(26.8)$ \\
Concomitant treatment not related to IBD, $n$ (\%) & $14(34.1)$ \\
Comorbility, $n$ (\%) & $16(39)$ \\
Anxiety, $n$ (\%) & $6(14.6)$ \\
Depression, $n$ (\%) & $4(9.8)$ \\
Adherence to thiopurines, $n$ (\%) & $32(78)$ \\
Adherence to thiopurines rate, median (IQR) & $94(81-100)$ \\
Adherence to biologicals, $n$ (\%) & $11(100)$ \\
Mayo score at the beginning of the study (Oct 17), & $0(0-0)$ \\
median (IQR) & $33(12)$ \\
Age at diagnosis of IBD (years), mean (SD) & $1.79(0.58)$ \\
Azathioprine daily dose (mg/kg), mean (SD) & $1.17(0.29)$ \\
Mercaptopurine daily dose (mg/kg), mean (SD) & $3(2-3)$ \\
No tablets/day, median (IQR) &
\end{tabular}

EIM: extraintestinal manifestations. ADR: adverse drug reaction. IBD: inflammatory bowel disease. SD: standard deviation. IQR: interquartile range

Table 2. Analysis of the relationship between qualitative-quantitative variables and adherence

\begin{tabular}{llllll} 
Characteristics & NON- & ADHERENT & $p$ - & OR & $95 \mathrm{Cl}$ \\
& $\begin{array}{l}\text { ADHERENT } \\
(n=9)\end{array}$ & $(n=32)$ & value & & \\
& & & & & \\
\hline $\begin{array}{l}\text { Gender } \\
\text { Male, } n(\%)\end{array}$ & $6(66.7)$ & $23(71.9)$ & & & \\
& & & & & \\
\end{tabular}




\begin{tabular}{|c|c|c|c|c|c|}
\hline Female, $n(\%)$ & $3(33.3)$ & $9(28.1)$ & & & \\
\hline $\begin{array}{l}\text { Family history of IBD, } n \\
\text { (\%) }\end{array}$ & $0(0)$ & $8(25)$ & 0.164 & 0.727 & $0.59-0.896$ \\
\hline Smoking habit, $n$ (\%) & $1(11.1)$ & $3(9.4)$ & 1 & 0.828 & $0.075-9.074$ \\
\hline Appendectomy, n (\%) & $0(0)$ & $2(6.3)$ & 1 & 0.769 & $0.648-0.914$ \\
\hline Disease extension, $n(\%)$ & & & & & \\
\hline E1, proctitis & $1(11.1)$ & $1(3.1)$ & 0.395 & 0.258 & $0.015-4.591$ \\
\hline E2, left-sided colitis & $3(33.3)$ & $6(18.8)$ & 0.384 & 0.462 & $0.089-2.393$ \\
\hline E3, pancolitis & $5(55.6)$ & $25(78.1)$ & 0.217 & 2.857 & $0.601-13.586$ \\
\hline$E I M, n(\%)$ & $5(55.6)$ & $8(25)$ & 0.113 & 0.267 & $0.057-1.243$ \\
\hline Complications, $n$ (\%) & $0(0)$ & $5(15.6)$ & 0.568 & 0.75 & $0.621-0.906$ \\
\hline $\begin{array}{l}\text { ADR to thiopurines, } n \\
\text { (\%) }\end{array}$ & $1(11.1)$ & 7 (21.9) & 0.659 & 2.24 & $0.238-21.072$ \\
\hline $\begin{array}{l}\text { Concomitant treatment } \\
\text { related to IBD, } n(\%)\end{array}$ & $6(66.7)$ & $24(75)$ & 0.68 & 1.5 & $0.303-7.432$ \\
\hline 5-ASA treatment, $n$ (\%) & $6(66.7)$ & $24(75)$ & 0.68 & 1.5 & $0.303-7.432$ \\
\hline $\begin{array}{l}\text { Biological treatment, } n \\
\text { (\%) }\end{array}$ & $4(44.4)$ & $7(21.9)$ & 0.217 & 0.35 & $0.074-1.664$ \\
\hline $\begin{array}{l}\text { Concomitant treatment } \\
\text { not related to IBD, } n(\%)\end{array}$ & $1(11.1)$ & $13(40.6)$ & 0.131 & 5.474 & $0.609-49.168$ \\
\hline Comorbility, $n$ (\%) & $2(22.2)$ & $14(43.8)$ & 0.441 & 2.722 & $0.488-15.198$ \\
\hline Anxiety, $n(\%)$ & $0(0)$ & $6(18.8)$ & 0.309 & 0.743 & $0.611-0.903$ \\
\hline Depression, $n(\%)$ & $1(11.1)$ & $3(7.3)$ & 1 & 0.828 & $0.075-9.074$ \\
\hline $\begin{array}{l}\text { Relapses first year, } n \\
\text { (\%) }\end{array}$ & 1 (11.1) & $8(25)$ & 0.654 & 2.667 & $0.287-24.738$ \\
\hline $\begin{array}{l}\text { Relapses second year, } \\
n(\%)\end{array}$ & $2(22.2)$ & $5(15.6)$ & 0.637 & 0.648 & $0.103-4.075$ \\
\hline Relapses (total), n (\%) & $2(22.2)$ & $10(31.3)$ & 0.702 & 1.591 & $0.279-9.066$ \\
\hline $\begin{array}{l}\text { Mild relapse first year, } \\
n(\%)\end{array}$ & 1 (11.1) & $4(12.5)$ & 1 & 1.143 & $0.111-11.722$ \\
\hline
\end{tabular}




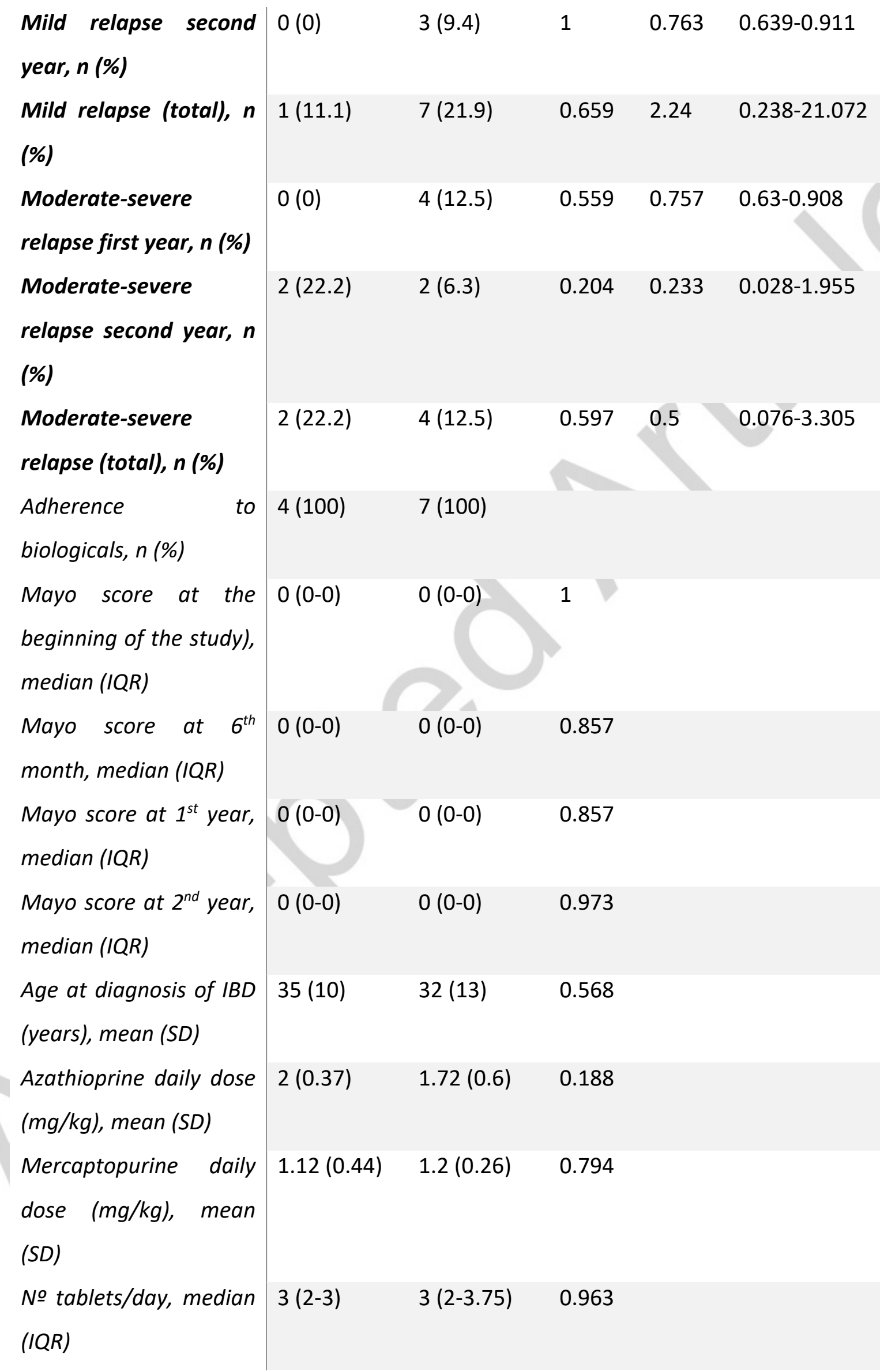


EIM: extraintestinal manifestations. ADR: adverse drug reaction. IBD: inflammatory bowel disease. SD: standard deviation. IQR: interquartile range

218 of 765 patients with UC at the IBD Unit of the Clinic University Hospital of Valencia were identified by ENEIDA as patients treated with thiopurines

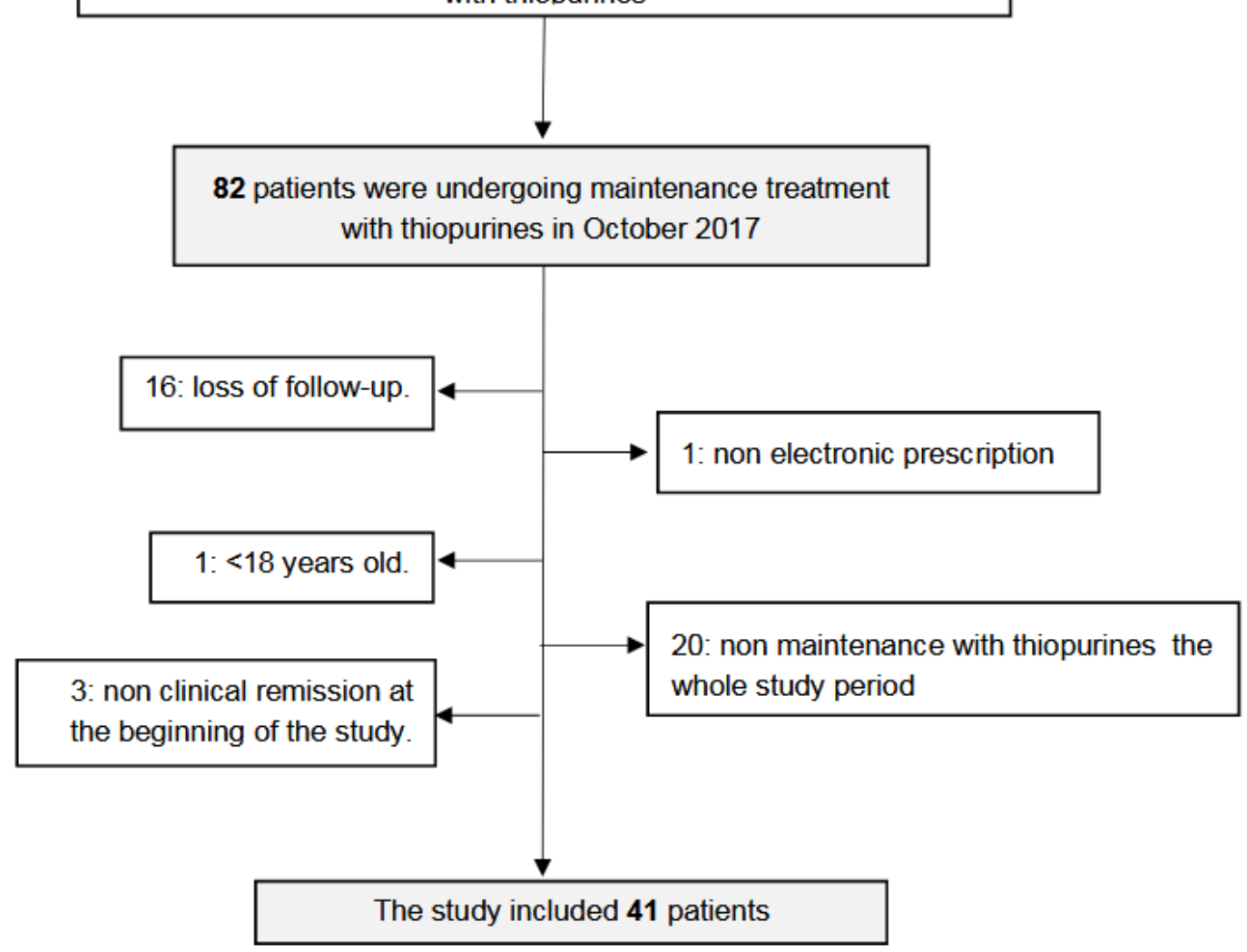

Figure 1. Flowchart of the patient selection process 


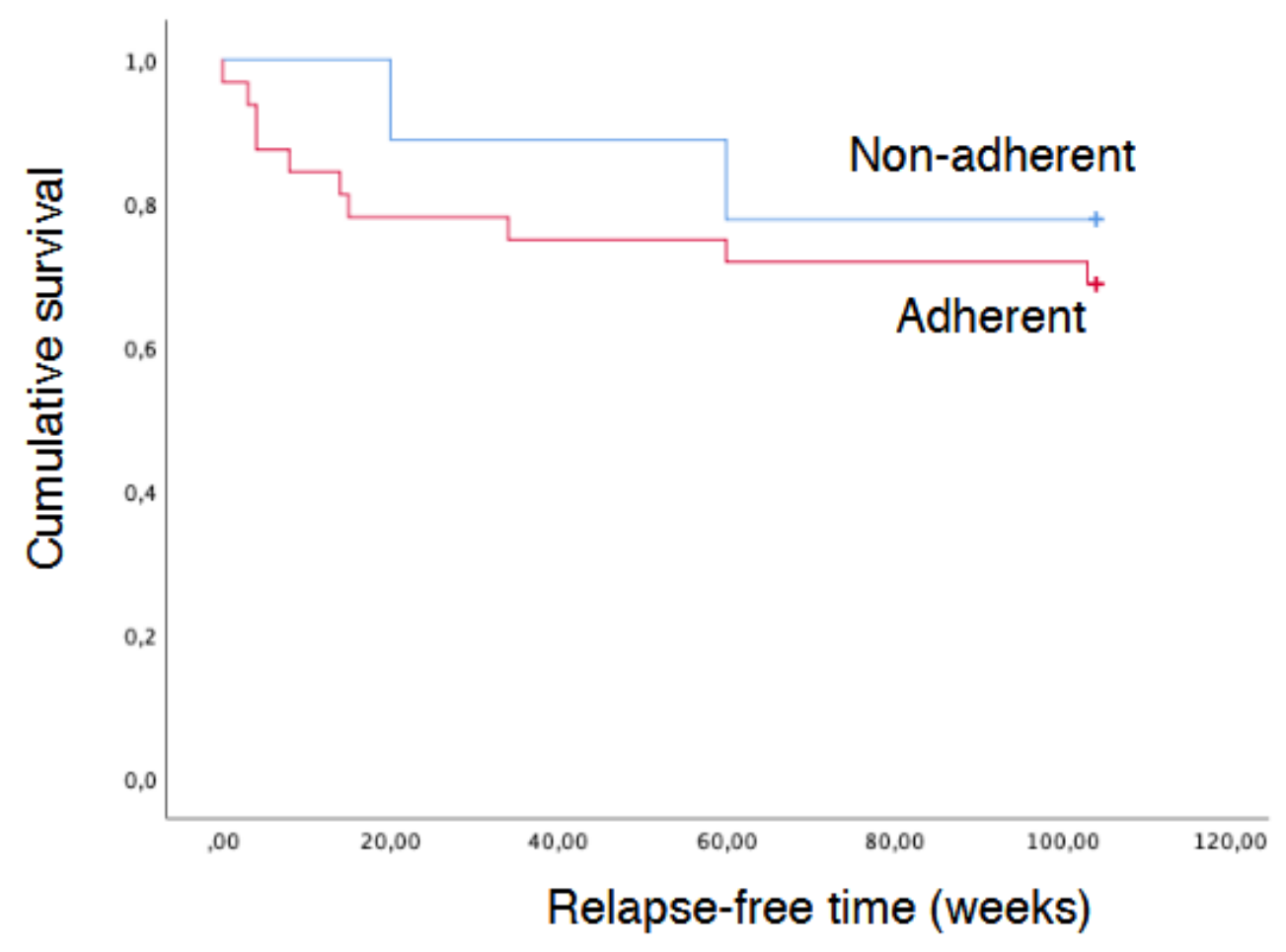

Figure 2. Time-to-relapse according to adherence 


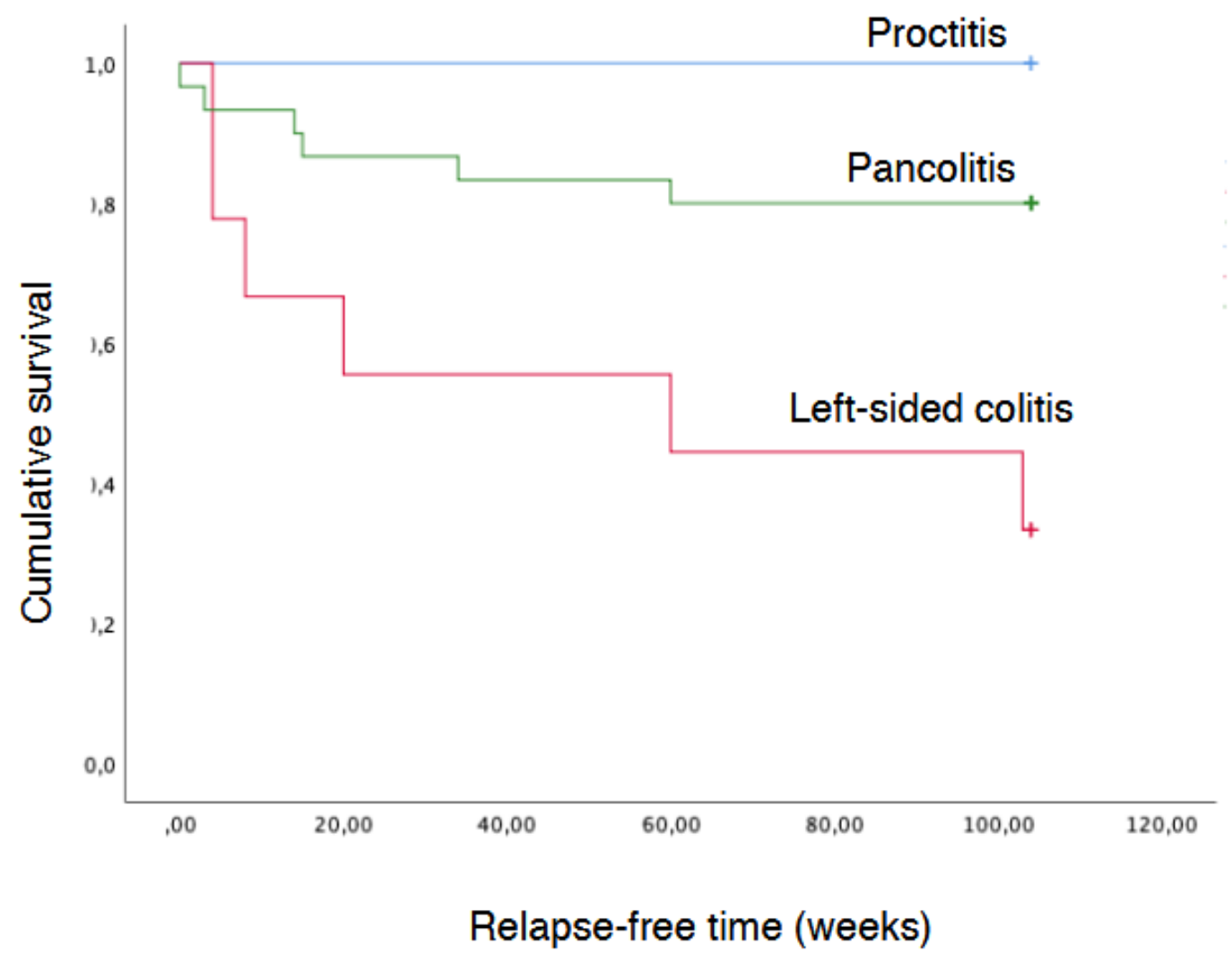

Figure 3. Time-to-relapse according to the disease extension 